\title{
Adjacent Display of Relevant Discussion Helps Resolve Confusion
}

\author{
MATIN YARMAND, UC San Diego, USA \\ SRISHTI PALANI, UC San Diego, USA \\ SCOTT KLEMMER, UC San Diego, USA
}

\begin{abstract}
Discussion fora of instructional videos contain previously-discussed questions and answers. These video comments can resolve many points of confusion for learners. However, finding relevant content in a separated discussion forum is challenging and disruptive to learning flow. This paper introduces Adjacent Display of Relevant Discussion (ADRD): it displays threaded comments in a panel adjacent to the video and dynamically updates the content of the panel based on the time of the video. In a between-subjects lab study ( $n=20)$, ADRD helped participants resolve confusion points, skim and read comments, and encouraged video interaction.
\end{abstract}

CCS Concepts: • Applied computing $\rightarrow$ Interactive learning environments; • Human-centered computing $\rightarrow$ Empirical studies in interaction design; Empirical studies in HCI.

Additional Key Words and Phrases: MOOC, confusion points, discussion forum, engagement, learning

ACM Reference Format:

Matin Yarmand, Srishti Palani, and Scott Klemmer. 2021. Adjacent Display of Relevant Discussion Helps Resolve Confusion. Proc. ACM Hum.-Comput. Interact. 5, CSCW1, Article 143 (April 2021), 11 pages. https: //doi.org/10.1145/3449217

\section{INTRODUCTION}

The embedded discussion fora in instructional videos facilitate teacher-student and student-student interactions [4] and support collaborative discourse for gaining knowledge [15, 19]. Students can use these fora to resolve their confusions, since often other users have asked similar questions and received responses from their instructors and peers. However, due to the chaotic and fragmented nature of the discussion fora, finding helpful comments can be challenging [10] and can hinder students' learning and motivation [7].

To assist learners in finding relevant content in the discussion fora, prior research has developed computational techniques to detect and cluster comments based on topics and potential confusions $[1,7,9]$. However, these studies do not address how and when to present discussion comments in order to provide helpful information without disrupting and overwhelming students.

To address these gaps, we introduce Adjacent Display of Relevant Discussion (ADRD) which dynamically retrieves relevant posts from the discussion fora. This interface retrieves comments according to their uniquely assigned time intervals, hand-labeled by the authors. ADRD also uses pause as a cue for confusion to present relevant content to the learner, analogous to a tutor who might provide clarifications when the student looks confused. ADRD places the discussion in-situ

Authors' addresses: Matin Yarmand, Design Lab, UC San Diego, La Jolla, CA, USA, myarmand@ucsd.edu; Srishti Palani, Design Lab, UC San Diego, La Jolla, CA, USA, srpalani@ucsd.edu; Scott Klemmer, Design Lab, UC San Diego, La Jolla, CA, USA, srk@ucsd.edu.

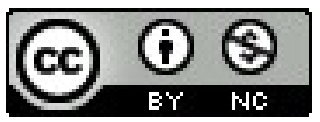

This work is licensed under a Creative Commons Attribution-NonCommercial International 4.0 License.

(C) 2021 Copyright held by the owner/author(s).

2573-0142/2021/4-ART143. https://doi.org/10.1145/3449217

Proc. ACM Hum.-Comput. Interact., Vol. 5, No. CSCW1, Article 143. Publication date: April 2021. 
of the video and enables users to follow both content simultaneously without having to repeatedly navigate between the video and forum (see Figure 1).

We report on a between-subjects lab study $(n=20)$ that investigated people's interaction with discussion comments in the baseline Khan Academy interface vs. ADRD. This study revealed that ADRD participants resolved more confusion points, and skimmed and read more comments, but received similar quiz scores to the baseline group. This work makes the following contributions:

(1) an interface that leverages discussion fora and learners' interactions to detect when and how to intervene with relevant information to help resolve learners' confusion, and

(2) qualitative and quantitative insights from a lab study that highlight the potential benefits of this approach in resolving confusion.

\section{RELATED WORK}

This section presents an overview of factors that can enhance learning in instructional videos, including the organization and design of discussion fora, and video interaction.

\subsection{Discussion Fora Promote Peer Learning}

Students use the discussion fora of instructional videos to ask for clarification from peers, share thoughts, and build a sense of togetherness $[7,16,36]$. They also collaboratively create knowledge [34]: this type of interaction relates to Communities of Practice $(\mathrm{CoP})$ which refers to a group of individuals with a common set of objectives [23]. Thomas [35] applied this framework to an online learning environment and demonstrated that participants promoted learning without the involvement of an expert and solely via peer interaction.

Students consult these fora to resolve confusions when watching videos [1]. Confusions, if unaddressed, lead to boredom, frustration, and eventually disengagement with the learning process [22]. Prolonged confusion can lead to poorer student achievement [24] and even increased dropout [41]. Just-in-time detection of confusion and providing relevant content can help resolve learners' confusion and enhance learning. Similar to a tutor, a helpful discussion interface can detect learners' confusion points and provide relevant information. This informs our first two design goals: intuitively detecting confusion, and presenting contextually-relevant discussion.

\subsection{Effective Display of Discussion Is Challenging}

When designing video discussion interfaces it is challenging to simultaneously facilitate user engagement and deep conversations without disrupting the video. Popular instructional video platforms contain a separate, static comment section either below the video (e.g. Khan Academy), or on a separate page (e.g., edX and Coursera). The disconnect between the video and discussion can make it difficult to grasp information from both sources [21]. Synchronous Overlay, such as Danmaku and CommenTV, displays temporally-anchored comments on the video screen [17,39]. While Danmaku comments enhance user engagement, they obscure the video content underneath [27, 40]. Also, the fragmented nature and poor addressivity of Danmaku comments hinder interactions and deep conversations [27]. This is especially important in a learning environment where collaborative discourse contributes to knowledge gain $[15,19]$. Presenting threaded comments adjacent to the video can provide rich discussions in-situ of the video without obscuring the video content. Our third design goal (i.e., easy navigability of discussion fora) incorporates these characteristics.

\subsection{Organization of Discussion Lowers Learning Barriers}

Automatic topic-based clustering of comments organizes chaotic and unstructured discussion fora $[3,7,9,33,38]$. Clustering helps instructors navigate the forum and provide systematic responses [7],

Proc. ACM Hum.-Comput. Interact., Vol. 5, No. CSCW1, Article 143. Publication date: April 2021. 
and assists students find posts of interest that address their confusion [2]. For instance, Wise et al. [38] developed a classification model to identify whether discussion posts related to the course content and found that content-related posts have distinct linguistic features.

Other classification techniques relied on sentiment analysis, and more specifically, students' confusion. Agrawal et al.[1] developed YouEDU that automatically detects the confusion from discussion comments and suggests relevant video snippets. Similarly, Zhu et al. [44] analyzed the discussion forum of a programming course, identified threads that expressed frustration, and categorized them based on course content. Aggregation of similar content from the discussion fora can assist learning, especially when the comments relate to students' confusion. This motivates our second design goal: presenting contextually-relevant discussion.

\subsection{Video Engagement Improves Learning and Satisfaction}

Higher engagement with the video (e.g., pausing and re-watching) enhances learning and satisfaction: Zhang et al. [43] found that students in interactive video environments can control their pace of learning to achieve higher attentiveness and learning outcomes. Besides, interactivity with video enhances learners' perceived satisfaction $[26,43]$ which is a strong predictor of retention in online courses [14]. Inspired by the ICAP framework [5], Active Viewing [8] explains the benefits of engagement with video content: active learners utilize affordances in their environment to better support their learning [32].

To enhance video interactions and navigability, LectureScape [18] and ToolScape [11] summarize user interaction data, such as a timeline histogram and a set of frequent words. While these tools focus on students' video interaction, they do not consider the discussion fora. Our first design goal proposes incorporating users' video interactions (i.e., pause) to provide relevant comments.

In conclusion, discussion fora can help learning by highlighting and resolving potential confusions. However, this is an under-utilized resource because navigating and interacting with discussion fora can be challenging. In this paper, we explore the research question: how can we leverage discussion fora and learners' interactions to detect when and how to intervene with information to help resolve learners' confusion, encourage engagement and promote learning?

\section{DESIGN OF ADJACENT DISPLAY OF RELEVANT DISCUSSION}

This section describes the design goals of ADRD, as displayed in Figure 1:

\subsection{Intuitively detecting confusion}

ADRD uses pause as a cue for learners' confusion. Once a user pauses or seeks to a different time in the video, the discussion presents comments that relate to the content at the particular time of the video (Figure 1a). This is consistent with students' usual behaviour, who when confused, pause the video and consult learning resources [25].

\subsection{Presenting contextually relevant discussion}

To ensure that the discussion is felicitous, and not overwhelming, ADRD maps and presents discussion posts to relevant content of the video. The discussion panel updates comments based on when the video pauses (see Figure 1(b)). In this study, we hard-coded the mapping of discussion to the video. To anchor comments to specific parts of the video, we consulted the taxonomy of referent types in video comments [42]. We determined these references based on three types of video content in the taxonomy (i.e., Visual, Auditory, and Conceptual). Table 1 depicts the definition of each type in our context with an example comment. For each comment, we then assigned the time period that the content appears in the video. We stored these time intervals with the accompanying 


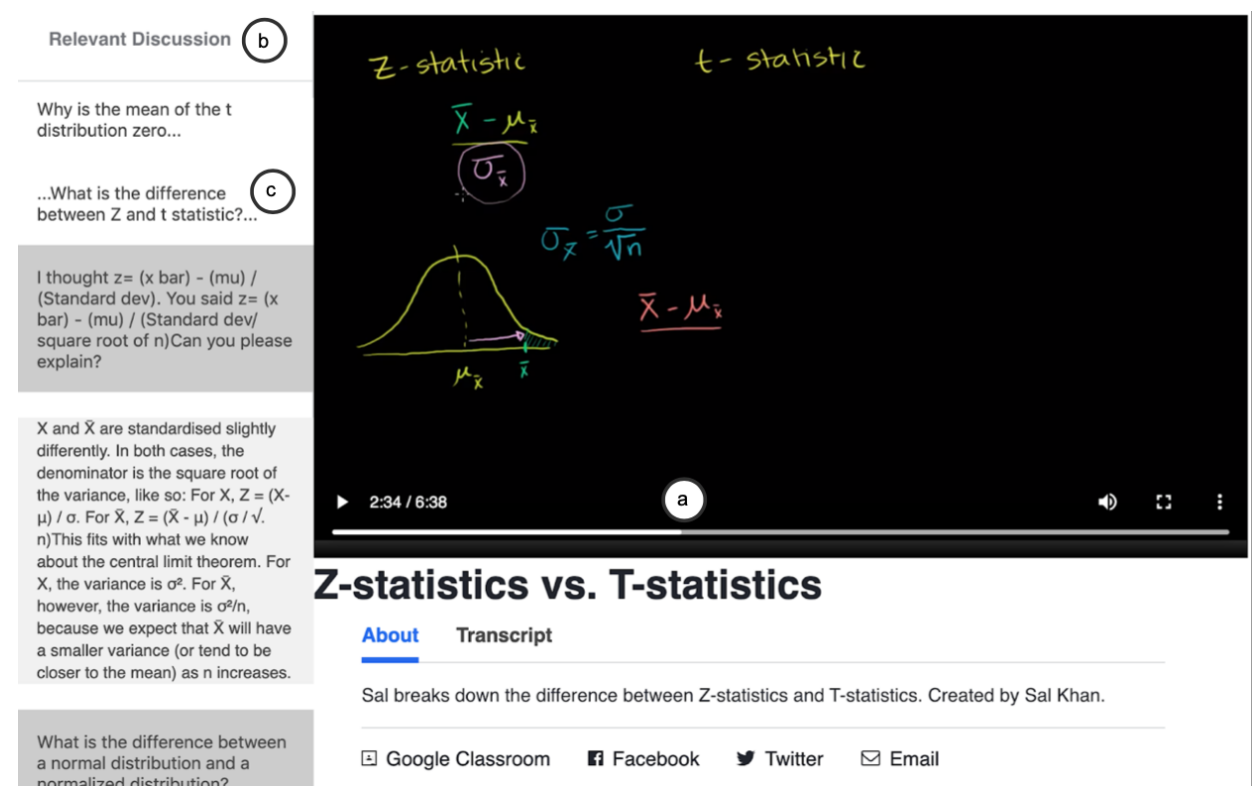

Fig. 1. ADRD displays discussion comments adjacent to the instructional video and updates the content when user pauses: (a) Pausing the video displays comments that relate to the time of the video. (b) The Relevant Discussion panel presents relevant comments. (c) Each question is a snippet. When clicked on, it expands to display the entire thread. Questions and answers have different colors to assist skimming.

comments. The discussion panel displays comments once user pauses at a time that is within the assigned intervals. For each comment, we added a short snippet to enable faster skimming. Prior work discovered strong correlation between confusion and question [1,37]. So to capture the main confusion in each comment, we extracted the embedded question: we located the first occurrence of question mark and used the preceding sentence.

\begin{tabular}{lll}
\hline Category & Definition & Example Comment \\
\hline Visual & Visible content on the blackboard, & Does he draw the t-distribution slightly \\
& such as words, symbols, and drawings & right skewed on purpose?
\end{tabular}

Table 1. The three types of video content that guided assigning time intervals to Khan Academy comments. The example comments appear in the video titled Z-statistics vs. T-statistics.

\subsection{Designing discussion fora to be easily navigable}

The left-hand side panel enables reading comments synchronously without obscuring the video content. The panel initially presents each question as a short snippet: this enables participants to 
quickly skim and assess whether the comment is relevant to their information need. To read more of the comment and its response thread, users can click on the snippet, as demonstrated in Figure 1(c). The original post has a different color than its following threaded comments to further help navigability.

\section{METHOD}

20 students at a western research university (10 female, 9 male, and 1 non-binary; 8 undergraduate and 12 graduate students) participated in a between-subject experiment. This study presented participants with a six minute video from Khan Academy, titled "Z-statistics vs. T-statistics". The video features Khan-style tablet drawing, a particularly engaging style of instructional video [12].

\subsection{Study Procedure}

Participants spent 35 minutes on the study. The study began by explaining the procedure to the participants and providing them with an informed consent form. A pre-task quiz assessed participants' prior knowledge. The quiz comprised of seven multiple-choice questions: four questions were the same as viewers' confusions in the video discussion and three were from the Khan Academy quiz associated with the video. The participants had seven minutes to complete the pre-quiz.

Once they finished the pre-quiz, participants were randomly assigned to one of two conditions: ADRD or Forum Comments (i.e., Khan Academy interface). Participants were counterbalanced based on their academic status (i.e., undergraduate or graduate degree) to account for the potential difference in their problem-solving skills. They then proceeded to watch the video and interact with the interface in the allocated 12 minutes. The screen is recorded. Since many students report taking notes while learning from online videos [13], participants were given a blank sheet of paper. Researchers removed these notes in the later stages of the study. To incentivize participants for higher engagement, they were informed that the top $25 \%$ would receive a $\$ 5$ gift card.

After watching the video, participants completed the same seven quiz questions that they answered in the beginning, in a randomized order. Lastly, to gain insight into participants' cognitive processes, they performed a retrospective think-aloud: they watched the recorded screens of the main task and verbally expressed their thought process.

\subsection{Measures}

The study measured the following six variables:

- Number of comments skimmed: The number of comments in which the participant read the question but not the replies. This was identified by the participant in the retrospective think-aloud, or in the screen recording when user paused longer or moved the cursor and highlighted a section.

- Number of comments read: The number of comments in which the participant read both the question and replies. This is a subset of the previous measure.

- Number of confusion points: The number of questions or concepts that the participant identified as confusing in the retrospective think-aloud.

- Number of confusion points resolved: The number of concepts that the participant found confusing but then the confusion was resolved, as identified in the retrospective think-aloud. Since resolution of confusion is a type of learning, this is the first measure of learning.

- Normalized change in Quiz Scores: Since recalling of information is a type of learning, measuring normalized change between pre- and post-task quiz scores is the second measure of learning [28]. The use of pre-quiz/post-quiz is a common method to assess learning gain [6, 31].

- Number of video interactions: The frequency of interacting with the video in terms of three engagement metrics: pausing, seeking, and replaying. Pausing and replaying are two active 
behaviours in the Active Viewing framework [8]. Seeking to a time point further in the video was also a noticeable type of video engagement in this study.

\section{RESULTS}

This section compares ADRD interface to Forum Comments and presents differences in how users resolve confusion, skim and read comments, interact with the video, and learn the content.

\subsection{ADRD Resolved More Points of Confusion}

As displayed in Figure 2, ADRD participants resolved $65 \%$ of the confusions that they encountered while watching the video (11 out of 17 confusions), whereas the Forum comments users indicated that only five out of 27 of their confusions (19\%) got resolved. Our Chi-square test with Yates' continuity correction revealed that ADRD significantly increased the resolution of confusion $\left(X^{2}(1\right.$, $N=20)=7.72, p=.005)$.

Participants pointed out the benefits of ADRD with respect to identifying and resolving their confusions. For instance, P20 said "having the comments right there was really helpful in understanding what others found confusing about this part of the video too". Another ADRD user also said "in many places, there were some questions that I wouldn't have thought of if not for the prompts and the site" (P5).

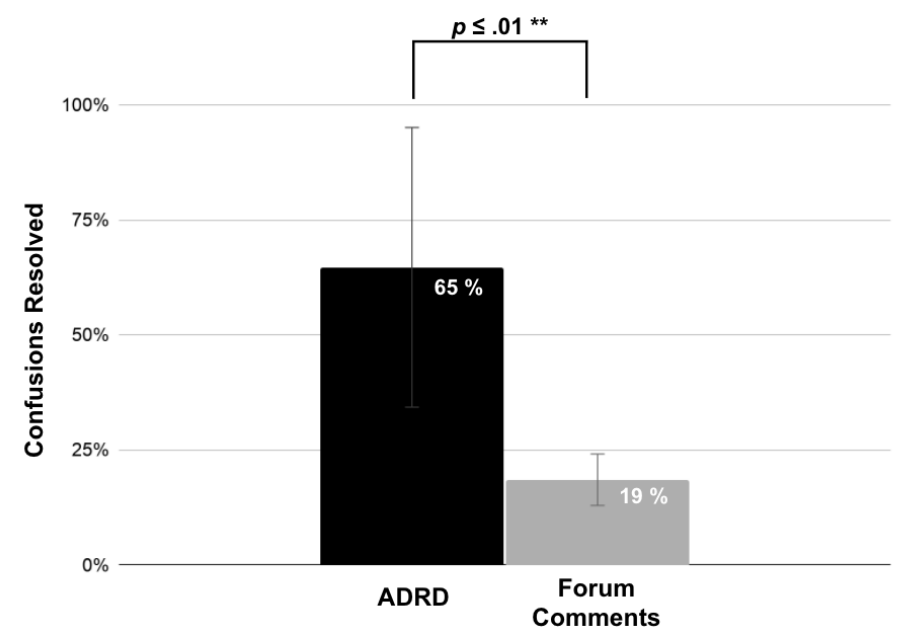

Fig. 2. ADRD participants significantly resolved more points of confusion than the Forum Comments users $(p<.01)$.

\subsection{ADRD Showed Insignificant Impact on Quiz Performance}

Our analysis of the normalized change in pre- and post-quiz scores revealed similar means across the two groups; ADRD users achieved an average of $0.23(\sigma=0.39)$ and Forum Comments gained $0.18(\sigma=0.33)$ learning. An unpaired t-test revealed an insignificant difference between the two groups $\left(t_{9}=-0.31, p=.76\right)$.

Qualitatively, ADRD participants indicated unique facets of learning as they interacted with the system: P20 said "I learned not only what was in the video, but also got to see and learn from the discussion around different parts of the video". P11 also mentioned "from the discussion, I could learn 
stuff that wasn't in the video". Another ADRD participant used the discussion to learn selective parts of the video:

Having watched the video and reading through the stuff that other people have said, my brain picks up on the things that he is saying. It helps knowing what to pay attention to versus having to pay attention to everything. (P18)

\subsection{ADRD Helped Participants Skim and Read More Discussions}

As reported in Figure 3, ADRD participants skimmed roughly three times as many comments ( $\mu=11$, $\sigma=48.44)$ as the participants in the Forum Comments $\left(\mu=3.6, \sigma=15.82, t_{9}=-2.91, p=.009\right)$. Participants in the ADRD group also read significantly more comments $(\mu=5.2, \sigma=17.07)$ than Forum Comments users $\left(\mu=2, \sigma=5.78, t_{9}=-2.12, p=.05\right)$.

Forum Comments participants struggled to find the relevant discussion, and hence, they felt discouraged to look further in the comments sections: for instance, P10 said "I was trying to see [in the comments section] if anyone else talked about the variables. I gave up and just looked at the graph [in the video]." On the other hand, P8, an ADRD participant who skimmed and read 18 and 12 comments respectively, associated the ease of skimming to how the system displays discussion, explaining that "the shortened questions were a good indicator of what you would expect underneath".

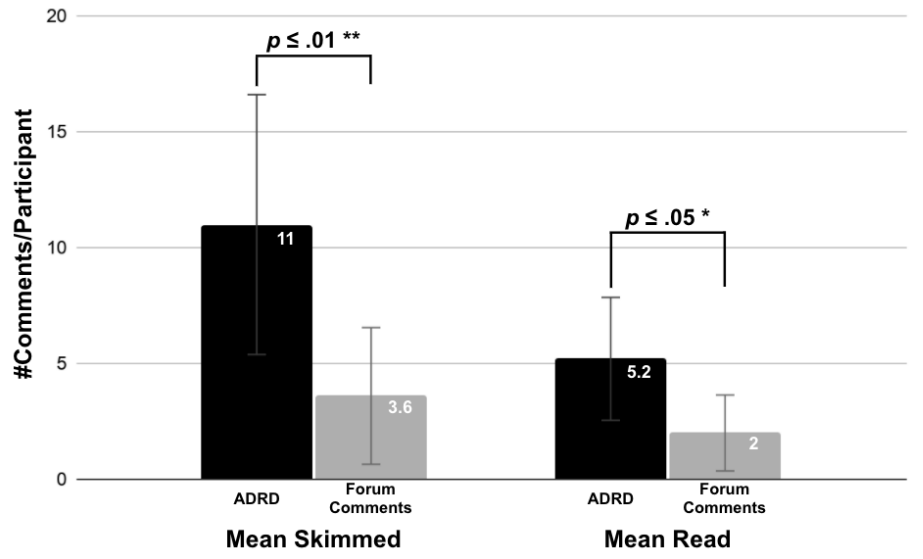

Fig. 3. ADRD Participants skimmed 11 comments on average compared to 3.6 comments for Forum Comments users $(p<.01)$. Besides, ADRD and Forum Comments participants read an average of 5.2 and 2 comments, respectively $(p=.05)$.

\subsection{ADRD Encouraged Video Interactions}

To assess participants' interactions with the video, this analysis considers three metrics: replay, pause, and seek (to video parts that are ahead). ADRD users replayed the video $(\mu=1.5, \sigma=20.4)$ more than the Forum Comments users with marginal significance $\left(\mu=0.4, \sigma=2.4, t_{9}=-1.99, p=.06\right)$. Participants re-watched parts of the video to "re-listen to a complicated term" ( $P 3$, a Forum Comments user), "revisit comments that had vanished" (P5, an ADRD user), or "to re-watch missed explanation" (P8, also an ADRD user).

While ADRD users paused $(\mu=2.4, \sigma=5.82)$ and sought to a different part of the video $(\mu=2.8$, $\sigma=20.62$ ) more than Forum Comments users (pauses: $\mu=1.7, \sigma=0.9, t_{9}=-0.85, p=0.40$; seeks: $\mu=1.5$, $\sigma=14.06, t_{9}=-0.69, p=0.49$ ), this difference was not statistically significant at the $p<.05$ level. However, 
ADRD participants pointed out how the system encourages pause, since it prompts displaying new comments in the Relevant Discussion panel. For instance, P5 reflected on the ease of finding new discussion in ADRD which promoted pausing the video frequently:

One good thing about things on the side is that people have this inertia to pause the video and go look about it. They always think OK I'll take a look about it later and then they forget. So, if it's just to the side of it, it actually helps. It doesn't take a lot of effort to look at them [points at the comments in the dynamic discussion panel].

Similar to pause, reading new comments motivated ADRD participants to seek to other parts of the video, such as $P 8$ who said "I didn't have a particular question. I just wanted to see what people were asking about. I am scrubbing to see if anything new pops up." P18, another ADRD participant, leveraged comments in further parts of the video to strengthen their understanding of the content:

I wanted to see if things start to click a little better knowing what everybody else has been asking, knowing the scope of everything [the narrator] is getting ready to say which helps me to solidify the material.

\section{DISCUSSION}

This section reflects on the increased resolution of confusion points and video engagement in ADRD, and the insignificant difference in quiz scores between ADRD and Forum Comments.

\subsection{How ADRD Users Resolved More Confusion?}

While ADRD resolved 65\% of participants' confusion points, those assigned to Forum Comments only resolved $19 \%$ of their confusions. The following can explain the difference. First, the display of comments (i.e., shortened questions and color difference between the main post and replies) helped participants skim and read more discussion. Reading more relevant comments can solidify understanding and expose students to a diverse set of knowledge. Contrary to ADRD, Forum Comments participants often avoided seeking similar discussions once they found relevant comments at the top of the static forum.

Second, formulating a good question often requires substantial domain knowledge: to ask good questions, one must know enough to know what is not known [29]. Clearly, not all students can ask good questions. However, having easy access to previous discussions can help resolve students' confusions, especially for those who might not be able to adequately express their questions. As presented in section 5.1, one of the participants expressed a similar sentiment: "in many places, there were some questions that I wouldn't have thought of if not for the prompts and the site" (P5).

\subsection{Why Resolution of More Confusion Points Did Not Increase Learning Gain?}

This study measures learning using resolution of confusion and normalized change in pre- and post-quiz scores. While ADRD participants resolved significantly more confusion points than those in the Forum Comments group, normalized change stayed the same. Limited scale might have influenced our measure of normalized change in quiz scores, both in terms of the number of participants and the number of questions. Similar studies that achieved significant difference in learning gains were conducted in larger scales; for instance Pickering [31] recruited 49 participants across an entire semester and administered four tests, each containing ten questions. While a lab study provided an understanding of people's interaction with the interface, a large scale study can better assess the potential learning benefits.

To evaluate learning gains more robustly, another consideration is to ensure that the questions asked in the pre- and post-quiz cover a diverse set of learning goals. While we diversified the sources of quiz questions (e.g., Khan Academy end-of-lesson questionnaire and discussion comments), these 
questions did not evenly contain all the learning content in the video. Selecting a more diverse set of questions might reveal differences in learning gains.

\subsection{How ADRD Encouraged Video Interaction?}

As stated in section 5.4, ADRD can encourage video interaction in the form of pausing, re-watching, and seeking. These active behaviours can enhance user engagement and improve satisfaction, learning, and retention $[8,30,43]$. Besides, in online education students rely on peer-based interactions for motivation and learning [20]. All of the aforementioned behaviours prompt the discussion panel to display peer discussions. ADRD further enhances benefits of video interactions by making peer discussions more accessible (i.e., adjacent display) and navigable (i.e., shortened questions with a different color than replies).

\section{CONCLUSION AND FUTURE WORK}

This paper introduces $\mathrm{ADRD}$, an interface for retrieving relevant discussion and displaying these comments adjacent to the video. ADRD contains a dynamic discussion panel which updates every time the user pauses or seeks to a different part of the video. The panel presents snippets of discussion posts for faster skimming. A between-subjects lab study $(n=20)$ revealed that ADRD can significantly resolve more points of confusion and help users skim and read more discussion.

This work has a number of limitations that can be addressed in future studies. First, while we only recruited 20 participants, a large-scale experiment can validate the benefits of ADRD. Second, the monetary incentivization of $5 \$$ for the top $25 \%$ was not ecologically valid. Future learning-based studies can be incorporated into class curricula where students are more strongly motivated to participate. Third, this study manually assigns time intervals to the video comments. This hand-labeling process is time-intensive and inefficient to scale. Future studies can automate or crowd-source labeling. Fourth, follow-up studies on ADRD can explore other facets of distance learning, such as remote peer-interactions (e.g., asking and answering questions while viewing the same video) and spatial mapping of discussion to the video content.

\section{REFERENCES}

[1] Akshay Agrawal, Jagadish Venkatraman, Shane Leonard, and Andreas Paepcke. 2015. YouEDU: addressing confusion in MOOC discussion forums by recommending instructional video clips. (2015).

[2] Omaima Almatrafi and Aditya Johri. 2018. Systematic review of discussion forums in massive open online courses (MOOCs). IEEE Transactions on Learning Technologies 12, 3 (2018), 413-428.

[3] Thushari Atapattu and Katrina Falkner. 2016. A framework for topic generation and labeling from MOOC discussions. In Proceedings of the Third (2016) ACM Conference on Learning@ Scale. 201-204.

[4] Christopher G Brinton, Mung Chiang, Shaili Jain, Henry Lam, Zhenming Liu, and Felix Ming Fai Wong. 2014. Learning about social learning in MOOCs: From statistical analysis to generative model. IEEE transactions on Learning Technologies 7, 4 (2014), 346-359.

[5] Michelene TH Chi. 2009. Active-constructive-interactive: A conceptual framework for differentiating learning activities. Topics in cognitive science 1, 1 (2009), 73-105.

[6] Henri G Colt, Mohsen Davoudi, Septimiu Murgu, and Nazanin Zamanian Rohani. 2011. Measuring learning gain during a one-day introductory bronchoscopy course. Surgical endoscopy 25, 1 (2011), 207-216.

[7] Yi Cui and Alyssa Friend Wise. 2015. Identifying content-related threads in MOOC discussion forums. In Proceedings of the Second (2015) ACM Conference on Learning@ Scale. 299-303.

[8] Samuel Dodson, Ido Roll, Matthew Fong, Dongwook Yoon, Negar M Harandi, and Sidney Fels. 2018. An active viewing framework for video-based learning. In Proceedings of the Fifth Annual ACM Conference on Learning at Scale. 1-4.

[9] Aysu Ezen-Can, Kristy Elizabeth Boyer, Shaun Kellogg, and Sherry Booth. 2015. Unsupervised modeling for understanding MOOC discussion forums: a learning analytics approach. In Proceedings of the fifth international conference on learning analytics and knowledge. 146-150.

[10] Oliver Ferschke, Iris Howley, Gaurav Tomar, Diyi Yang, Yu Liu, and Carolyn Penstein Rosé. 2015. Fostering discussion across communication media in massive open online courses. International Society of the Learning Sciences, Inc.[ISLS]. 
[11] Krzysztof Z Gajos, Juho Kim, Shang-Wen Li, Carrie J Cai, and Robert C Miller. 2014. Leveraging video interaction data and content analysis to improve video learning. (2014).

[12] Philip J Guo, Juho Kim, and Rob Rubin. 2014. How video production affects student engagement: An empirical study of MOOC videos. In Proceedings of the first ACM conference on Learning@ scale conference. 41-50.

[13] Negar M Harandi, Farshid Agharebparast, Luis Linares, Samuel Dodson, Ido Roll, Matthew Fong, Dongwook Yoon, and Sidney Fels. 2018. Student video-usage in introductory engineering courses. Proceedings of the Canadian Engineering Education Association (CEEA) (2018).

[14] Kate S Hone and Ghada R El Said. 2016. Exploring the factors affecting MOOC retention: A survey study. Computers \& Education 98 (2016), 157-168.

[15] Carol Hostetter. 2013. Community matters: Social presence and learning outcomes. Fournal of the Scholarship of Teaching and Learning 13, 1 (2013), 77-86.

[16] Matthew B Hoy. 2014. MOOCs 101: an introduction to massive open online courses. Medical reference services quarterly 33, 1 (2014), 85-91.

[17] Jee Yeon Hwang, Pol Pla i Conesa, Henry Holtzman, and Marie-José Montpetit. 2012. CommenTV: A time-sensitive social commenting system for audiovisual content. In 2012 IEEE Consumer Communications and Networking Conference (CCNC). IEEE, 84-88.

[18] Juho Kim, Philip J Guo, Carrie J Cai, Shang-Wen Li, Krzysztof Z Gajos, and Robert C Miller. 2014. Data-driven interaction techniques for improving navigation of educational videos. In Proceedings of the 27th annual ACM symposium on User interface software and technology. 563-572.

[19] Jungjoo Kim, Yangyi Kwon, and Daeyeon Cho. 2011. Investigating factors that influence social presence and learning outcomes in distance higher education. Computers \& Education 57, 2 (2011), 1512-1520.

[20] Chinmay Kulkarni, Koh Pang Wei, Huy Le, Daniel Chia, Kathryn Papadopoulos, Justin Cheng, Daphne Koller, and Scott R Klemmer. 2013. Peer and self assessment in massive online classes. ACM Transactions on Computer-Human Interaction (TOCHI) 20, 6 (2013), 1-31.

[21] Rodrigo Laiola Guimarães, Pablo Cesar, and Dick CA Bulterman. 2012. " Let me comment on your video" supporting personalized end-user comments within third-party online videos. In Proceedings of the 18th Brazilian symposium on Multimedia and the web. 253-260.

[22] Reed W Larson and Maryse H Richards. 1991. Boredom in the middle school years: Blaming schools versus blaming students. American journal of education 99, 4 (1991), 418-443.

[23] Jean Lave, Etienne Wenger, et al. 1991. Situated learning: Legitimate peripheral participation. Cambridge university press.

[24] Yunsup Lee, Rimas Avizienis, Alex Bishara, Richard Xia, Derek Lockhart, Christopher Batten, and Krste Asanović 2011. Exploring the tradeoffs between programmability and efficiency in data-parallel accelerators. In Proceedings of the 38th annual international symposium on Computer architecture. 129-140.

[25] Nan Li, Łukasz Kidziński, Patrick Jermann, and Pierre Dillenbourg. 2015. MOOC video interaction patterns: What do they tell us? In Design for teaching and learning in a networked world. Springer, 197-210.

[26] Shu-Sheng Liaw. 2008. Investigating students' perceived satisfaction, behavioral intention, and effectiveness of e-learning: A case study of the Blackboard system. Computers \& education 51, 2 (2008), 864-873.

[27] Xiaojuan Ma and Nan Cao. 2017. Video-based evanescent, anonymous, asynchronous social interaction: Motivation and adaption to medium. In Proceedings of the 2017 ACM Conference on Computer Supported Cooperative Work and Social Computing. 770-782.

[28] Jeffrey D Marx and Karen Cummings. 2007. Normalized change. American Journal of Physics 75, 1 (2007), 87-91.

[29] Naomi Miyake and Donald A Norman. 1979. To ask a question, one must know enough to know what is not known. Journal of verbal learning and verbal behavior 18, 3 (1979), 357-364.

[30] Pam Northrup. 2001. A framework for designing interactivity into web-based instruction. Educational technology 41, 2 (2001), 31-39.

[31] James D Pickering. 2017. Measuring learning gain: Comparing anatomy drawing screencasts and paper-based resources. Anatomical sciences education 10, 4 (2017), 307-316.

[32] Ido Roll and Philip H Winne. 2015. Understanding, evaluating, and supporting self-regulated learning using learning analytics. Fournal of Learning Analytics 2, 1 (2015), 7-12.

[33] Lorenzo A Rossi and Omprakash Gnawali. 2014. Language independent analysis and classification of discussion threads in Coursera MOOC forums. In Proceedings of the 2014 IEEE 15th International Conference on Information Reuse and Integration (IEEE IRI 2014). IEEE, 654-661.

[34] G Stahl, T Koschmann, and D Suthers. 2006. CSCL: An Historical Perspective on Computer-supported collaborative learning. Cambridge handbook of the learning sciences (2006), 409-426.

[35] Angela Thomas. 2005. Children online: Learning in a virtual community of practice. E-Learning and Digital Media 2, 1 (2005), 27-38. 
[36] Matthew JW Thomas. 2002. Learning within incoherent structures: The space of online discussion forums. fournal of Computer Assisted Learning 18, 3 (2002), 351-366.

[37] Nancy Wilson. 1989. Learning from confusion: Questions and change in reading logs. The English fournal 78, 7 (1989), 62-69.

[38] Alyssa Friend Wise, Yi Cui, Wanqi Jin, and Jovita Vytasek. 2017. Mining for gold: Identifying content-related MOOC discussion threads across domains through linguistic modeling. The Internet and Higher Education 32 (2017), 11-28.

[39] Qunfang Wu, Yisi Sang, and Yun Huang. 2019. Danmaku: A New Paradigm of Social Interaction via Online Videos. ACM Transactions on Social Computing 2, 2 (2019), 1-24.

[40] Qunfang Wu, Yisi Sang, Shan Zhang, and Yun Huang. 2018. Danmaku vs. forum comments: Understanding user participation and knowledge sharing in online videos. In Proceedings of the 2018 ACM Conference on Supporting Groupwork. 209-218.

[41] Diyi Yang, Miaomiao Wen, Iris Howley, Robert Kraut, and Carolyn Rose. 2015. Exploring the effect of confusion in discussion forums of massive open online courses. In Proceedings of the second (2015) ACM conference on learning@ scale. 121-130.

[42] Matin Yarmand, Dongwook Yoon, Samuel Dodson, Ido Roll, and Sidney S Fels. 2019. " Can you believe [1: 21]?!" Content and Time-Based Reference Patterns in Video Comments. In Proceedings of the 2019 CHI Conference on Human Factors in Computing Systems. 1-12.

[43] Dongsong Zhang, Lina Zhou, Robert O Briggs, and Jay F Nunamaker Jr. 2006. Instructional video in e-learning: Assessing the impact of interactive video on learning effectiveness. Information \& management 43, 1 (2006), 15-27.

[44] Joyce Zhu, Jeremy Warner, Mitchell Gordon, Jeffery White, Renan Zanelatto, and Philip J Guo. 2015. Toward a domain-specific visual discussion forum for learning computer programming: An empirical study of a popular MOOC forum. In 2015 IEEE Symposium on Visual Languages and Human-Centric Computing (VL/HCC). IEEE, 101-109.

Received June 2020; revised October 2020; accepted December 2020 